\title{
Biological activities and potential therapeutic applications of functional oligosaccharides
}

\begin{abstract}
There is an increased interest on researching the functions of oligosaccharides due to their biological activities offer positive effects on our health. The functional oligosaccharides have been widely applied in various branches, such as pharmacological supplements and food ingredients. They are widely found in plants, algae, bacteria, and higher fungi. Pectic oligosaccharides have been regarded as a new kind of prebiotics capable of exerting a number of health-promoting effects, it can protect colonic cells against Shiga toxins, prevent the adhesion of uropathogenic microorganisms, etc. Milk oligosaccharides can promote the growth of the beneficial bacterial flora in the intestines and develop resistance to bacterial and viral infections. Keeping in this view, this article summarized here to discuss the sources, benefit effects, and synthesis of the functional oligosaccharides.
\end{abstract}

Keywords: oligosaccharides, functional properties, milk oligosaccharides, chemo enzymatic synthesis, HMOs

\author{
Volume 5 Issue I - 2017 \\ Shanshan Lai,' Chengfeng Yang, ${ }^{1,5}$ Sidi Liu, ${ }^{4}$ \\ Sinan Yuan, 'Yuanyuan Liu,' Xin Yan,' Chao \\ Zhao $^{1,2,3}$ \\ 'College of Food Science, Fujian Agriculture and Forestry \\ University, China \\ ${ }^{2}$ Fujian Province Key Laboratory for the Development of \\ Bioactive Material from Marine Algae, Quanzhou Normal \\ University, China \\ ${ }^{3}$ Department of Chemistry, University of California, USA \\ ${ }^{4}$ College of Life Science, Yantai University, China \\ ${ }^{5}$ College of Food Science and Nutritional Engineering, China \\ Agricultural University, China
}

Correspondence: Chao Zhao, College of Food Science, Fujian Agriculture and Forestry University, Fuzhou 350002, China, Tel 86-59I-83530197, Fax 86-59I-83530197, Email zhchao@live.cn

Received: August 28, 2017 | Published: October 26, 2017
Abbreviations: HMOs, human milk oligosaccharides; OPME, onepotmultienzyme; GlyK, glycokinase; NucT, nucleotidyltransferase; GlyT, glycosyltransferase; PpA, pyrophosphatase

\section{Introduction}

Modern consumers attach great importance to their personal health. They not only pursue tasty and attractive foods, but also safe and healthy foods. Therefore, more and more scientists have tried to seek ways to taste well and hold healthy by eating nutritionally designed foods. Functional food components have a wide range of applications and academic significance. Such as the non-digestible carbohydrates can better service health and reduce the risk of diseases, particularly the functional oligosaccharides. Functional oligosaccharides exist important physiological and physicochemical properties that contribute to improving consumer health; hence, their use as food ingredients has increased rapidly. ${ }^{1}$ Despite since the 1950s was known that selected carbohydrates stimulate the growth of beneficial bacteria in the intestine, their use for the production of health promoting functional foods start to be subject of interest many years later, when intensive research on the role of intestinal microbiota in many aspects of health highlighted the potential biological activities of those carbohydrates, especially prebiotics. ${ }^{2}$ Prebiotics can promote the growth of beneficial bacteria, particularly bifid bacteria species. ${ }^{3}$ According to extensive scientific research, functional oligosaccharides continue to stimulate great interest and exist potential future value. There is a commercial opportunity for functional oligosaccharides that devote to constructing a healthy diet, and for foods directly targeted to certain risk groups as well.

\section{Definition and sources of functional oligosaccharides}

According to the IUPAC-IUBMB Joint Commission on Biochemical Nomenclature, oligosaccharides is a kind of naturally occurring carbohydrates that are made up of 3-10 monosaccharide units, linear or branched, linked by $\alpha$-and/or $\beta$-glycosidic linkages. At present or under development, carbohydrates, whose monosaccharide units are glucose, fructose, galactose and/or xylose, are identified as the main kinds of functional oligosaccharides. ${ }^{4}$ Functional oligosaccharides are intermediate between monosaccharide and polysaccharides and are well known as prebiotics, due to they benefit the host by selectively stimulating beneficial bacteria in the intestine, especially bifid bacteria species. ${ }^{1,3,5}$ The best known functional oligosaccharides include isomaltooligosaccharides, glucooligosaccharide, fructooligosaccharide, maltitol, soybeanoligosaccharides and xylooligosaccharides. ${ }^{6}$

Functional oligosaccharides originate from various sources. Seaweeds and plants are the richest sources of functional oligosaccharides. ${ }^{7}$ Meanwhile, pectic oligosaccharides are produced by pectin depolymerization. Pectins are ramified heteropolymers made up of a linear backbone of $\alpha(1-4)$-linked D-galacturonic acid units. Citrus peels and apple pulp contain the most pectin contents, sugar beet pulp, peels of mango or peach and pumpkin pulp also have high pectin contents. ${ }^{8}$ Human milk contains very high concentrations of a structurally diverse group of more than two hundred different complex sugars called human milk oligosaccharides (HMOs), which are unconjugated complex glycans. HMOs are made up of five monosaccharide building blocks: galactose, glucose, $\mathrm{N}$-acetylglucosamine, fucose and $\mathrm{N}$-acetyl-neuraminic acid. They act as many important roles, such as serve as anti-adhesives, human milk prebiotics, and anti-microbials. ${ }^{9}$ Chitosan and its derivatives can be shown in various fields such as food, cosmetics, biomedicine, and agriculture. Chitosan oligosaccharides have high solubility and low viscosity in water, particularly at neutral $\mathrm{pH}$. They have amount of benefits for human beings, for example, they can decrease blood cholesterol, control of high blood pressure, protect from infections, and improve the anti-cancer property. Wheat, maize bran, sugarcane 
bagasse, rice and other plant sources can extract feruloylated oligosaccharides. Carrageenans are extracted from marine red algae. ${ }^{3}$

\section{Health benefit application of functional oligosaccharides}

There is no doubt that the gastrointestinal tract is covered by bacteria, particularly anaerobic bacteria. Although most of these bacteria are benign to the host, there are some of bacteria are pathogenic and may cause chronic disorders. ${ }^{10}$ Functional oligosaccharides can only be consumed by a limited number of bacteria due to their chemical structure. The bifidobacteria and lactobacilli are considered as the only microorganisms able to benefit the host's health. For example, the bifidobacteria can stabilize the gut mucosal barrier, modulate the immune response and intestinal microbiota, prevent traveler's diarrhea in children and so on. ${ }^{11}$ Isomaltooligosaccharide are reported that can increase cecal Bifidobacterium population in young broiler chickens. ${ }^{12}$ Furthermore, functional oligosaccharides can significantly modify the colonic microflora, because these oligosaccharides could inhibit the growth of putrefactive and pathogenic bacteria, such as soybean meal oligosaccharides, may promote competitive exclusion of potential pathogens. Some functional oligosaccharides act as a growth factor for these microorganisms which obtain human milk.

Different strains of lactobacilli compose healthy vaginal flora which is considered to protect the vagina against pathogenic microorganisms through different mechanisms. However, microbes may grow excessively when lactobacilli are reduced or absent and thus causing disorders. Indeed, vaginal flora imbalance results in the most urogenital infections. Among the functional oligosaccharides (prebiotics, fructooligosaccharides and glucooligosaccharides) can promote proliferation of lactobacilli avoiding the urogenital infections against pathogenic microorganisms.

Nowadays, type II diabetes and obesity can be seemed all over the world, especially the modern Western society. Some researchers have proved that the type II diabetes and obesity are related to the dietary fiber intake. The dietary fiber can control the release of glucose with time in the blood, management of diabetes mellitus and obesity. ${ }^{13}$ Functional oligosaccharides are considered as the important source of dietary fibers. ${ }^{14}$ It can modulate satiety, glucose or lipid metabolism, and hypertension when intake of them. The high functional oligosaccharides food has been discovered that can reduce postprandial blood glucose and insulin responses and improve the overall blood glucose and lipid concentrations. The arabinoxylan oligosaccharides can against type II diabetes; it can decrease postprandial glucose levels and insulin response and increase postprandial ghrelin. ${ }^{1}$ Dietary fiber has a ability that bind with bile acids and prevents its reabsorption in the liver thereby, inhibit cholesterol synthesi. ${ }^{15,16}$ The functional oligosaccharides, such as Gum Arabic has applications to reduce plasma glucose and serum cholesterol due to its intrinsic glycaemic index is near zero, and it is not ingested in the small intestine.

Functional oligosaccharides also play an important role in bettering diarrhea, it may be directly associated with the possible effect of bifidobacteria. ${ }^{16}$ Some researchers also found that functional oligosaccharides may improve the absorption of water and electrolytes in the small intestine, thereby leading to a reduction in the incidence of diarrhea and its duration. ${ }^{1}$ The functional oligosaccharides of various origins (viruses, bacteria, plants and fungi) have been demonstrated to exert potent immunomodulatory activities, ${ }^{17}$ and the use of functional oligosaccharides, such as prebiotic functional food ingredients have a ability that to escape digestion and absorption in the upper part of the intestine and reach the lower part where they should induce fermentation and selective stimulation of the growth and activity of intestinal bacteria associated with health. ${ }^{18}$ Functional oligosaccharides also show that they can prevent cancers and tumors effectively, because they could act against toxicity and carcinogenesis include the reduction of chemical adsorption in relation with physicochemical capacity to bind chemicals, physiologic and mechanical effects to enhance fecal passage and bulking in the gastrointestinal tract. ${ }^{1}$ The use of functional oligosaccharides in diet plays a vital part in managing dyslipidemia. ${ }^{19}$ Optimal intake of functional oligosaccharides can reduce the risk of blood pressure and many other cardiovascular diseases. ${ }^{20}$

Several minerals (zinc, copper, selenium, magnesium and iron) are benefit for our health. Although they have many health benefits for us, functional oligosaccharides and their derivatives (chitosan) may influence the absorption of minerals. For example, chitosan may arrest the absorption of calcium. As we all know, calcium can promote the growth of the bones and teeth, and it is required for proper functioning of the heart and brain. The deficiency of calcium can cause osteoporosis and the fiber can balance the level of calcium.

\section{The synthetic methods of functional oligosaccharides}

According to the above description, it is no doubt that functional oligosaccharides have numerous beneficial effects for our bodies. Therefore, we need to find the methods for obtaining synthetic oligosaccharides to provide enough of such functional oligosaccharides for scientific research or clinical applications. There are two methods to produce the synthetic oligosaccharides. They are chemical or chemo-enzymatic synthesis. The chemical method is challenging because the reactions should be carried out under harsh conditions. What's more, large quantities of reagents and organic solvents are often toxic. However, chemo-enzymatic synthesis method can overcome these challenges. It can be applied in nearly some complex oligosaccharides. ${ }^{21}$ The use of enzymes in the synthesis of oligosaccharides has attracted a lot of interest. Glycosyltransferases and glycosidases involve in oligosaccharide synthesis in prokaryotes and eukaryotes. Glycosyltransferase-catalyzed enzymatic and chemo-enzymatic syntheses are considered to be effective ways for producing oligosaccharides. Enzymatic glycosylation occur stereoand regioselectively generally under mild conditions and therefore circumventing the tedious protection/deprotection steps. Functional enzymes enable the large-scale synthesis of difficult-to-produce saccharide linkages and complex molecules. It has been reported that an efficient one-pot multienzyme fucosylation system used for the gram-scale synthesis of lacto-N-fucopentaose I (Figure 1). The syntheses offer the unique advantage of flexibility, it can reduce the substrate costs for in vitro production of fucosylated carbohydrates and what's more, large-scale cost-effective production of HMOs may be conducted using whole cell systems. In a word, enzymes are highly efficient and flexible on the substrates and therefore often result in great yields of oligosaccharide products. ${ }^{3}$

\section{Discussion}

Current methods for obtaining synthetic oligosaccharides are chemo-enzymic method and chemical method (Figure 1). Chemoenzymic synthesis has priority compare to chemical synthesis. There are many advantages by using chemo-enzymic method. For example, some complex oligosaccharides and derivatives with 3-11 
monosaccharides units have been reported by preparative-scale and increased large-scale productions. The development of one-pot multienzyme-catalyzed syntheses can reduce the substrate costs for in vitro production of fucosylated carbohydrates. Through using the whole cell systems, cost-effective large-scale production of HMOs may be conducted. Although the chemo-enzymotic method has some advantages over its chemical counterpart, there are also some disadvantages cannot be ignored. One disadvantage to the chemo-enzymic method is identifying active glycosyltransferases and glycosidases by recombinant expression systems, but it is difficult to obtain good expression levels in recombinant expression systems. Such as the proteins expressed May due to misfolding in Escherichia coli systems and many proteins require post-translational modifications for activities in eukaryotes. ${ }^{3}$ Raising the knowledge on this issue can improve the production of such functional carbohydrates in glycoscience.

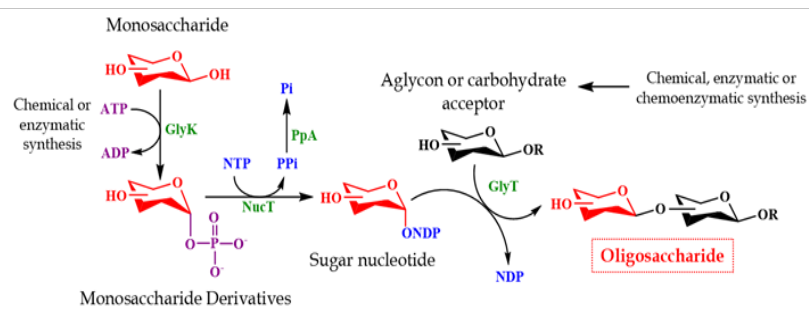

Figure I The simplest routes for one-pot multienzyme (OPME) chemoenzymatic synthetic strategy for producing oligosaccharide from a monosaccharide. GlyK, glycokinase; NucT, nucleotidyltransferase; GlyT, glycosyltransferase; PpA, pyrophosphatase. Modified with permission from. ${ }^{3}$

\section{Conclusion}

Functional oligosaccharides play a critical role in preventing inflammatory processes, reducing diabetes, obesity and cardiovascular risks, modulating the gut flora and affecting different gastrointestinal activities. In modern society, there are an increasing number of people who is often sedentary, and have a diet high in saturated fat and sugar, and low in fruit, vegetables and fiber. Functional oligosaccharides may relieve the symptoms which are caused by their bad habits. In this mini-review, we focus on the knowledge about functional oligosaccharides have an effect on intestinal microflora, diabetes, obesity and cardiovascular risks. Although a few major advances in carbohydrate synthesis have been covered, the current state of knowledge about the function of complex oligosaccharides is scarce. Raising knowledge on this topic it is expected to contribute to the synthesis of new oligosaccharides with enhanced target-specific functional properties offer new research and business opportunities. And there will be a great progress in food and pharmacological industry.

\section{Acknowledgements}

This work was financially supported by Fujian Province Key Laboratory for the Development of Bioactive Material from Marine Algae grant (2017FZSK05), National Natural Science Foundation of Fujian Province, China (2016J06009) and Key Project of Fuzhou Municipal Bureau of Science and Technology (2017-N-36). The project was also supported by FAFU grant KXb16011A.

\section{Conflicts of interest}

The author declares no conflict of interest.

\section{References}

1. $\mathrm{Xu} \mathrm{Q}$, Chao YL, Wan QB. Health benefit application of functional oligosaccharides. Carbohyd Polym. 2009;77(3):435-441.

2. Moreno FJ, Corzo N, Montilla A, et al. Current state and latest advances in the concept, production and functionality of prebiotic oligosaccharides. Current Opinion in Food Science. 2017;13:50-55.

3. Zhao C, Wu YJ, Liu XY, et al. Functional properties, structural studies and chemo-enzymatic synthesis of oligosaccharides. Trends Food Sci Tech. 2017;66:135-145.

4. Mussatto SI, Mancilha IM. Non-digestible oligosaccharides: A review. Carbohyd Polym. 2007;68(3):587-597.

5. Kunz C, Rudloff S. Health promoting aspects of milk oligosaccharides. Int Dairy J. 2006;16(11):1341-1346.

6. Chen HL, Lu YH, Lin J, et al. Effects of fructooligosaccharide on bowel function and indicators of nutritional status in constipated elderly men. Nutr Res. 2000;20(12):1725-1733.

7. Van Laere KM, Hartemink R, Bosveld M, et al. Fermentation of plant cell wall derived polysaccharides and their corresponding oligosaccharides by intestinal bacteria. J Agr Food Chem. 2000;48(5):1644-1652.

8. Gullon B, Gomez B, Martinez Sabajanes M, et al. Pectic oligosaccharides: Manufacture and functional properties. Trends Food Sci Tech. 2013;30(2):153-161

9. Bode L. The functional biology of human milk oligosaccharides. Early Hum Dev. 2015;91(11):619-622.

10. Rollo F, Luciani S, Marota I, et al. Persistence and decay of the intestinal microbiota's DNA in glacier mummies from the Alps. J Archaeol Sci. 2007;34(8):1294-1305.

11. Matamoros Fernandez LE. Introduction to ion trap mass spectrometry: Application to the structural characterization of plant oligosaccharides. Carbohyd Polym. 2007;68(4):797-807.

12. Thitaram SN, Chung CH, Day DF, et al. Isomaltooligosaccharide increases cecal bifidobacteria population in young broiler chickens. Poult Sci. 2005;84(7):998-1003.

13. Bennett N, Greco DS., Peterson ME, et al. Comparison of a low carbohydrate-low fiber diet and a moderate carbohydrate-high fiber diet in the management of feline diabetes mellitus. J Feline Med Surg. 2006;8(2):73-84.

14. De Alcantara PHN, Martim L, Silva CO, et al. Purification of a betagalactosidase from cotyledons of Hymenaea courbaril L. (Leguminosae). Enzyme properties and biological function. Plant Physiol Bioch. 2006;44(11-12):619-627.

15. Mussatto SI, Mancilha IM. Non-digestible oligosaccharides: A review. Carbohyd Polym. 2007;68(3):587-597.

16. Mourao JL, Pinheiro V, Alves A, et al. Effect of mannan oligosaccharides on the performance, intestinal morphology and cecal fermentation of fattening rabbits. Animal Feed Science and Technology. 2006;126(12):107-120.

17. Surenjav U, Zhang LN, Xu XJ, et al. Effects of molecular structure on antitumor activities of (1 leads to 3)- $\beta$-D-glucans from different Lentinus edodes. Carbohyd Polym. 2006;63(1):97-104. 
18. Arakane Y, Zhu QS, Matsumiya M, et al. Properties of catalytic, linker and chitin-binding domains of insect chitinase. Insect Biochem Molec. 2003;33(6):631-648.

19. Perry JC, Dalmeida V, Souza FG, et al. Consequences of subchronic and chronic exposure to intermittent hypoxia and sleep deprivation on cardiovascular risk factors in rats. Respir Physiol Neurobi. 2007;156(3):250-258.
20. Bodi V, Sanchis J, Lopez Lereu MP, et al. Evolution of 5 cardiovascular magnetic resonance-derived viability indexes after reperfused myocardial infarction. Am Heart J. 2007;153(4):649-655.

21. Zhao C, Wu YJ, Yu H, et al. The one-pot multienzyme (OPME) synthesis of human blood group $\mathrm{H}$ antigens and a human milk oligosaccharide (HMOS) with highly active Thermosynechococcus elongatus a1-2fucosyltransferase. Chem Comm. 2016;52(20):3899-3902. 